Sharif University of Technology
Scientia Iranica
SCIENTIA $\quad \begin{gathered}\text { Transactions } D: \text { Computer Science ES Engineering and Electrical Engineering } \\ \text { http://scientiairanica.sharif.edu }\end{gathered}$

\title{
Iterative method for simultaneous sparse approximation
}

\author{
S. Sadrizadeh, Sh. Kianidehkordi, M. Boloursaz Mashhadi, and F. Marvasti* \\ Advanced Communication Research Institute (ACRI), Electrical Engineering Department, Sharif University of Technology, Tehran,
} Iran.

Received 12 November 2017; received in revised form 22 July 2018; accepted 29 October 2018

\section{KEYWORDS}

Simultaneous sparse approximation; Iterative method; Joint recovery.

\begin{abstract}
This paper studies the problem of Simultaneous Sparse Approximation (SSA). This problem arises in many applications that work with multiple signals maintaining some degree of dependency, e.g., radar and sensor networks. We introduce a new method towards joint recovery of several independent sparse signals with the same support. We provide an analytical discussion of the convergence of our method, called Simultaneous Iterative Method (SIM). In this study, we compared our method with other group-sparse reconstruction techniques, namely Simultaneous Orthogonal Matching Pursuit (SOMP) and Block Iterative Method with Adaptive Thresholding (BIMAT), through numerical experiments. The simulation results demonstrated that SIM outperformed these algorithms in terms of the metrics Signal to Noise Ratio (SNR) and Success Rate (SR). Moreover, SIM is considerably less complicated than BIMAT, which makes it feasible for practical applications such as implementation in MIMO radar systems.

(C) 2019 Sharif University of Technology. All rights reserved.
\end{abstract}

\section{Introduction}

Sparse signal processing has recently been exploited in various fields of communication, because sparse signals can be approximated by only a few nonzero coefficients and, hence, sub-Nyquist sampling and Compressed Sensing (CS) [1-4]. The general CS problem is formulated as follows:

minimize $\|\mathbf{x}\|_{0}$

subject to $\|\mathbf{y}-\mathbf{A x}\|_{2} \leq \varepsilon$,

where $\mathbf{x}$ is the main sparse signal, $\mathbf{y}$ is the measurement vector, $\mathbf{A}$ is the sensing matrix, and $\|\mathbf{v}\|_{2} \leq \varepsilon$ with $\mathbf{v}$ representing the additive noise vector. Two main

*. Corresponding author.

E-mail addresses: ss.sadrizadeh@ee.sharif.edu (S. Sadrizadeh); shkianid@gmail.com (Sh. Kianidehkordi); boloursaz@ee.sharif.edu (M. Boloursaz Mashhadi); marvasti@sharif.edu (F. Marvasti)

doi: $10.24200 /$ sci. 2018.5564 .1347 models are considered in CS for reconstruction of sparse signals. Models with one measurement vector are referred to as Single Measurement Vector (SMV) models, while the other models with at least two measurement vectors are called Multiple Measurement Vector (MMV) models.

The problem investigated in MMV models, known as SSA, aims to jointly recover sparse representation of the measurement vectors. The SSA applications may be encountered in various fields such as sensor networks [5,6], Electroencephalography and Magnetoencephalography (EEG and MEG) [7], source localization [8], and distributed MIMO radar systems [9].

Mohammadi et al. [10] investigates the theory of MMV models. Some algorithms have been developed by extending the general SMV model into the MMV model to solve the SSA problems. Orthogonal Matching Pursuit (OMP) [11] as a greedy algorithm is one of the very first algorithms used for sparse recovery. At each iteration of this algorithm, the best local improvement to the current approximations is found in hope of obtaining a good overall solution. The extension of the 
OMP algorithm to the MMV paradigm, simultaneous OMP (SOMP), has been presented in [12-14].

The Iterative Method with Adaptive Thresholding (IMAT) algorithm was originally proposed for sparse signal reconstruction from missing samples [1521]. The Block Iterative Method with Adaptive Thresholding (BIMAT) [22] as an extension of IMAT is employed for block sparse recovery for distributed MIMO radar systems.

In this paper, we propose SIM for simultaneous reconstruction of jointly sparse signals from their missing samples.

\subsection{Paper overview}

The rest of this paper is structured as follows. In Section 2, we first provide the description of SSA model. Then the proposed method is introduced and its convergence is analyzed. Numerical experiments of our method in comparison with the SOMP algorithm are presented in Section 3. SIM is then demonstrated as a simple decoding algorithm for MIMO radar systems, and its performance is compared with BIMAT by means of simulation. Finally, the paper is concluded in Section 4.

\subsection{Notations}

Scalar variables, vectors, and matrices are denoted by italic lower-case, boldface lower-case, and boldface upper-case, respectively. The elements of a vector are denoted by subscripts, i.e., $x_{i}$ is the $i$-th element of the vector $\mathbf{x} . \quad|\mathbf{x}|$ calculates the absolute value of each entry of the vector $\mathbf{x}$. The pseudoinverse of matrix $\mathbf{A}$ is represented by $\mathbf{A}^{\dagger}$. Finally, the output of the thresholding operator $\mathrm{TH}(\mathbf{x}, \mathrm{thr})$ is defined as a diagonal matrix whose diagonal entries are determined as follows:

$$
\mathrm{TH}(\mathbf{x}, t h r)_{i i}= \begin{cases}1, & \left|x_{i}\right| \geq t h r \\ 0, & \left|x_{i}\right|<t h r\end{cases}
$$

\section{The proposed method}

\subsection{Problem statement}

In this section, we provide the formulation of SSA problems. Assume that $\mathbf{x}^{1}, \mathbf{x}^{2}, \ldots, \mathbf{x}^{L}$ are $L$ equallength signals that share the same sparsity support in a specific transform domain. It is assumed that the nonzero coefficients of the original sparse signals have a Gaussian distribution [23]. These signals are randomly sampled in another domain by independent sampling masks $\mathbf{s}^{1}, \mathbf{s}^{2}, \ldots, \mathbf{s}^{L}$. These masks are binary and each element of them is generated independently based on a Bernoulli distribution, i.e., $\mathbf{s}_{n}^{i} \sim \operatorname{Bernoulli}(p), 1 \leq$ $i \leq L$, and $0 \leq p \leq 1$ is the sampling probability. The randomly sampled signals $\mathbf{y}^{1}, \mathbf{y}^{2}, \ldots, \mathbf{y}^{L}$ are derived as follows:

$$
y_{n}^{i}=s_{n}^{i} \times x_{n}^{i}
$$

The problem is to simultaneously reconstruct the original sparse signals from their randomly sampled versions by employing the additional information that the signals share a common support in a specific transform domain.

\subsection{Algorithm}

One can find the SIM algorithm in Algorithm 1. Let $n, m$ and $k$ denote the lengths of the original signal, the length of the observed vectors, and the sparsity number, respectively. Moreover, let $\mathbf{x}_{i}^{j}$ represent the reconstruction of the $j$-th signal after $i$-th iteration.

It should be noted that the measurement matrices, i.e., $\left[\mathbf{A}^{1}, \mathbf{A}^{2}, \ldots, \mathbf{A}^{L}\right]$ can be calculated by multiplying the transformation matrix, which maps the signals to their sparsity domain, and diagonal matrices whose diagonal entries are equal to the elements of $\mathbf{s}^{1}, \mathbf{s}^{2}, \ldots, \mathbf{s}^{L}$.

This algorithm gradually extracts the sparse components of the signals by thresholding the estimated signals iteratively. Each iteration involves two different steps of thresholding and projection. The thresholding step provides an approximation of the common support of the signals by hard-thresholding the summation of the absolute values of the approximated signals. The projection step projects each of the estimated signals onto the convex set defined by the support vector approximated in the previous step.

In this algorithm, $\lambda$ is the relaxation parameter and controls the convergence speed. The threshold value is decreased exponentially by $\beta e^{-\alpha(k-1)}$, where $k$ is the iteration number. The performance of the algorithm is not much affected if the choice of the

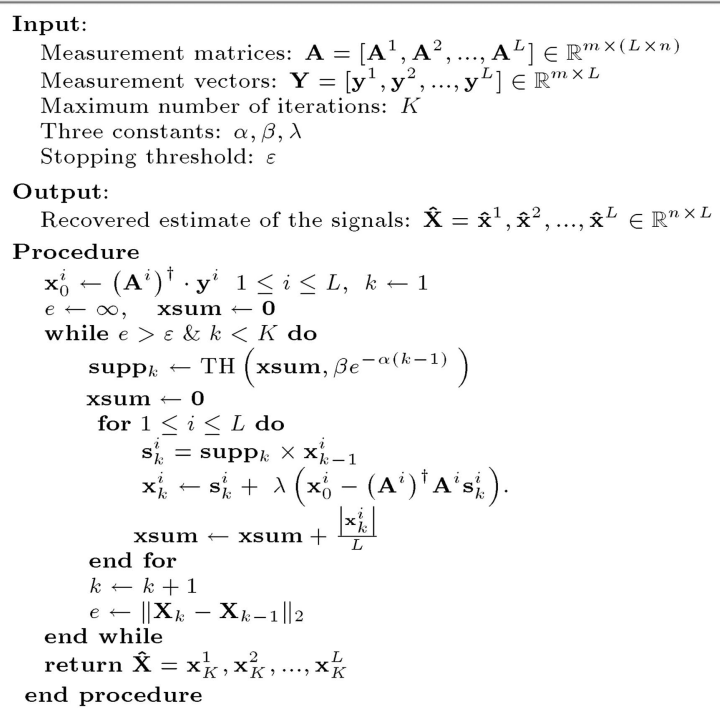

Algorithm 1. The pseudo-code of the proposed method: Simultaneous Iterative Method (SIM). 
parameters $\lambda, \beta$, and $\alpha$ is not optimum; however, these parameters are optimized empirically to achieve a faster convergence.

The algorithm is stopped when the maximum number of iterations is reached or the relative error between two consequent iterations is less than a threshold, i.e., $\left\|\mathbf{X}_{k}-\mathbf{X}_{k-1}\right\|_{2} \leq \varepsilon$, where $\mathbf{X}_{k}$ and $\mathbf{X}_{k-1}$ are the estimated signals in $k$-th and $(k-1)$-th iterations, respectively.

\subsection{Analytical discussion}

In [24], it is proved that under sufficient condition, the Iterative Method with Adaptive Thresholding (IMAT) converges to the sparsest solution to the random sample SMV problem. Now, we show that the probability of finding the support of signals with the SIM is more than that with the IMAT. Before proving this statement, we note the following points.

We assume that the original signals are sparse in the time domain, and each non-zero coefficient has a Gaussian distribution. Additionally, the signals are randomly sampled and polluted by additive white Gaussian noise in the frequency domain. Since the signals are estimated by line 16 of Algorithm 1 in each iteration, each coefficient of the estimated signals has a Gaussian distribution.

The distribution of the absolute value of a random variable with Gaussian distribution $N\left(0, \sigma^{2}\right)$ is halfnormal with the following probability density (PDF) and cumulative distribution ( $\mathrm{CDF})$ :

$$
\begin{aligned}
& P_{Y}(y ; \sigma)=\frac{\sqrt{2}}{\sqrt{\sigma^{2} \times \pi}} \exp \left(-\frac{y^{2}}{2 \times \sigma^{2}}\right) \quad y \geq 0, \\
& Q_{Y}(y ; \sigma)=\operatorname{erf}\left(\frac{y}{\sqrt{\sigma^{2} \times 2}}\right) .
\end{aligned}
$$

In the thresholding step of each iteration of our algorithm, the absolute values of the coefficients are calculated. Hence, these variables have half-normal distribution. We define the variable $z$ as the absolute value of the estimation of a non-zero coefficient of a signal and assume that it has variance $\sigma_{1}^{2}$ and mean $\mu_{1}$. Similarly, we define the variable $w$ associated with a zero coefficient of a signal and assume that its variance and mean are $\sigma_{0}^{2}$ and $\mu_{0}$, respectively. We also assume $\mu_{1}>\mu_{0}$ since the variance of the additive noise is small. Due to the fact that the means of $L$ signals is calculated for the SIM, the variables $z_{L}$ and $w_{L}$ can be defined as the mean of the variables mentioned above. ${ }_{2}$ The variance and the mean of $z_{L}$ and $w_{L}$ are $\frac{\sigma_{1}^{2}}{L}, \mu_{1}, \frac{\sigma_{0}^{2}}{L}$ and $\mu_{0}$, as a result of the independency of signals.

We prove the superiority of SIM over IMAT through Lemmas 1 and 2. As a general idea of these lemmas, according to the law of large numbers, the coefficients of the signal in the SIM get closer to their mean when $L$, the number of signals, goes toward infinity. In fact, the none-zero coefficients tend to $\mu_{1}$ and the zero coefficients tend to $\mu_{0}$. Therefore, it would be easier to find the non-zero coefficients of the original signals.

Lemma 1. If the number of signals $L$ satisfies the following inequality:

$$
L \geq \frac{\sigma_{0}^{2}}{\left(\varepsilon-\mu_{0}\right)\left(1-\operatorname{erf}\left(\frac{\varepsilon}{\sqrt{\sigma_{0}^{2} \times 2}}\right)\right)}>0
$$

then $P(w \geq \varepsilon) \geq P\left(w_{L} \geq \varepsilon\right), \forall \varepsilon \geq 2 \times \mu_{0}$, where $P$ is the probability sign. $w$ and $w_{L}$ are the variables representing the estimated zero coefficients of a signal in each iteration of IMAT and SIM, respectively.

Proof. As a result of $\varepsilon \geq 2 \times \mu_{0}$ and positivity of $w_{L}$, one can easily show the following equality:

$$
P\left(w_{L} \geq \varepsilon\right)=P\left(\left|w_{L}-\mu_{0}\right| \geq \varepsilon-\mu_{0}\right) .
$$

According to the Chebyshev's theorem, we have:

$$
P\left(\left|w_{L}-\mu_{0}\right| \geq \varepsilon-\mu_{0}\right) \leq \frac{\frac{\sigma_{0}^{2}}{L}}{\varepsilon-\mu_{0}} .
$$

If we define $Q_{w}(\varepsilon)$ as the $\mathrm{CDF}$ of $P(w)$, we get:

$$
P(w \geq \varepsilon)=1-Q_{w}(\varepsilon)=1-\operatorname{erf}\left(\frac{\varepsilon}{\sqrt{\sigma_{0}^{2} \times 2}}\right) .
$$

Since $1-\operatorname{erf}\left(\frac{\varepsilon}{\sqrt{\sigma_{0}^{2} \times 2}}\right) \geq 0$ and $\left(\varepsilon-\mu_{0}\right) \geq 0$, the lemma is proved for the number of signals specified by Relation (6).

Lemma 2. If the number of signals $L$ satisfies the following inequality:

$$
L \geq \frac{\sigma_{1}^{2}}{\left(\mu_{1}-\varepsilon\right)\left(\operatorname{erf}\left(\frac{\varepsilon}{\sqrt{\sigma_{1}^{2} \times 2}}\right)\right)}>0,
$$

then $P(z \geq \varepsilon) \leq P\left(z_{L} \geq \varepsilon\right), \forall \mu_{1} \geq \varepsilon \geq 2 \times \mu_{0}$, where $P$ is the probability sign and the estimated nonzero coefficients of a signal in each iteration of IMAT and SIM are denoted by $z$ and $z_{L}$, respectively.

Proof. It is not too difficult to derive the following inequality:

$$
P\left(z_{L} \geq \varepsilon\right) \geq 1-P\left(\left|z_{L}-\mu_{1}\right| \geq \mu_{1}-\varepsilon\right) .
$$

According to the Chebyshev's theorem, we have:

$$
P\left(\left|z_{L}-\mu_{1}\right| \geq \mu_{1}-\varepsilon\right) \leq \frac{\frac{\sigma_{1}^{2}}{L}}{\mu_{1}-\varepsilon} \Rightarrow
$$




$$
P\left(z_{L} \geq \varepsilon\right) \geq 1-\frac{\sigma_{1}^{2}}{L}
$$

If we define $Q_{z}(\varepsilon)$ as the $\mathrm{CDF}$ of $P(z)$, then we get:

$$
P(z \geq \varepsilon)=1-Q_{z}(\varepsilon)=1-\operatorname{erf}\left(\frac{\varepsilon}{\sqrt{\sigma_{1}^{2} \times 2}}\right)
$$

Since $\operatorname{erf}\left(\frac{\varepsilon}{\sqrt{\sigma_{1}^{2} \times 2}}\right) \geq 0$ and $\left(\mu_{1}-\varepsilon\right) \geq 0$ for $\mu_{1} \geq$ $\varepsilon \geq 2 \times \mu_{0}$, one can easily prove the lemma for the $L$ indicated in Relation (10).

Theorem 1. If the number of signals $L$ satisfies the following inequality:

$$
\begin{aligned}
\forall \mu_{1} & \geq \varepsilon \geq 2 \times \mu_{0}, \\
L & \geq \max \left(\frac{\sigma_{1}^{2}}{\left(\mu_{1}-\varepsilon\right)\left(\operatorname{erf}\left(\frac{\varepsilon}{\sqrt{\sigma_{1}^{2} \times 2}}\right)\right)},\right. \\
& \left.\frac{\sigma_{0}^{2}}{\left(\varepsilon-\mu_{0}\right)\left(1-\operatorname{erf}\left(\frac{\varepsilon}{\sqrt{\sigma_{0}^{2} \times 2}}\right)\right)}\right),
\end{aligned}
$$

then $\forall \mu_{1} \geq \varepsilon \geq 2 \times \mu_{0}$, and the probability of finding the support with SIM is higher than that with IMAT.

Proof. According to Lemma 1, if $L$ satisfies Relation (6), the probability of mistaking a zero coefficient for a support in SIM is less than that in IMAT. According to Lemma 2, if L satisfies Relation (10), the probability of finding a none-zero coefficient in SIM is higher than that in IMAT.

Therefore, if $L$ satisfies Relation (14), it is more probable to find the support with the SIM than with the IMAT.

One can find the minimum value of $L$ which satisfies Relation (14) as shown in Table 1. This table shows that the minimum number of signals $L$ that guarantees superiority of SIM over IMAT is very low; hence, there is no need to have a large number of signals to benefit from SIM.

Table 1. Minimum number of signals that satisfies Theorem 1.

\begin{tabular}{cccc}
\hline Input SNR $\mathbf{( d B )}$ & $\mathbf{1 0}$ & $\mathbf{2 0}$ & $\mathbf{5 0}$ \\
\hline$\mu_{0}$ & 0.25 & 0.079 & 0.0025 \\
$\mu_{1}$ & 0.5 & 0.5 & 0.5 \\
$\sigma_{0}^{2}$ & 0.1 & 0.01 & $10^{-5}$ \\
$\sigma_{1}^{2}$ & 0.18 & 0.09 & 0.08 \\
$\varepsilon$ & 0.5 & 0.2 & 0.011 \\
Minimum Value of $\boldsymbol{L}$ & $\mathbf{4}$ & $\mathbf{2}$ & $\mathbf{5}$ \\
\hline
\end{tabular}

\section{Simulation Results}

In each trial of our simulation, we generate $L$ number of $K$-sparse signals. We choose $K$ components out of $N=256$ randomly, and set them to a random number in the interval $[-1,1]$. Then, the noisy signals are random-sampled by a sampling rate of $M / N$. By the law of algebra, the number of samples needed to specify the sparsity profile of the signals is at least twice the sparsity number, hence $K \leq M /(2 \times N)$. We optimize the parameters of the algorithm in each trial.

Table 2 compares the average reconstruction SNR (dB) of three algorithms, namely IMAT, SIM and SOMP, for different density numbers and input SNRs. As observed in Table 2, the simultaneous reconstruction methods outperform IMAT, especially for higher sparsity numbers.

In the case of not knowing the density rate, SIM yields the best results both in low and high density rates as well as in noisier channels. In noiseless channels, the signal can be perfectly reconstructed by SIM when the sparsity number is small.

In the case of knowing the density rate, SOMP and SIM exhibit similar performances in all the cases. However, based on the results in Table 3, SIM outperforms SOMP in terms of the complexity measured by the run-time.

The success rates of IMAT, SIM, and SOMP algorithms for different density rates are depicted in Figure 1. A reconstruction is considered to be successful if the output SNR is more than 20dB. As seen in this figure, all curves experience a sudden knee-like fall as the density rate increases. This fall is considered as the boundary between successful and unsuccessful reconstruction. The simulation results reveal that for the SIM, success rate falls around $20 \%$ density rate, while the knee-like fall happens in $18 \%$ and $12 \%$ density rates for IMAT and SOMP, respectively. This indicates that SIM can successfully reconstruct the signals with high sparsity numbers in comparison with SOMP and IMAT algorithms.

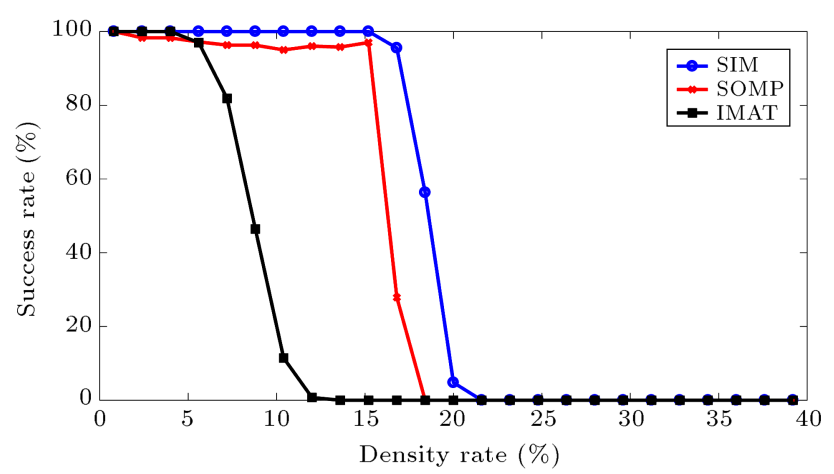

Figure 1. Success rate versus density rate (input SNR = $20 \mathrm{~dB}$, sampling rate $=25 \%, L=8$, and the sparsity number is unknown). 
Table 2. Reconstruction SNRs $(\mathrm{dB})$ of three different algorithms for different density rates $(K(\%))$ in the presence of noise $(\mathrm{SNR} 0=$ input $\mathrm{SNR}$, sampling rate $=25 \%, L=8)$.

\begin{tabular}{|c|c|c|c|c|c|c|c|c|c|c|c|c|c|c|c|c|c|c|}
\hline \multirow{3}{*}{$\begin{array}{c}\mathrm{SNR}_{0}(\mathrm{~dB}) \\
K(\%)\end{array}$} & \multicolumn{9}{|c|}{$K$ is Unknown } & \multicolumn{9}{|c|}{$K$ is Known } \\
\hline & \multicolumn{3}{|c|}{10} & \multicolumn{3}{|c|}{20} & \multicolumn{3}{|c|}{100} & \multicolumn{3}{|c|}{10} & \multicolumn{3}{|c|}{20} & \multicolumn{3}{|c|}{100} \\
\hline & 4 & 12 & 20 & 4 & 12 & 20 & 4 & 12 & 20 & 4 & 12 & 20 & 4 & 12 & 20 & 4 & 12 & 20 \\
\hline IMAT & 19.7 & 0.5 & -1.3 & 31.4 & 0.7 & -1.8 & 94.3 & 0.9 & -2.1 & 19.9 & 2.1 & 1.3 & 31.4 & 2.2 & -0.5 & 94.6 & 1.4 & -1.9 \\
\hline SIM & 23.2 & 16.1 & 1.8 & 31.1 & 25.4 & 4.6 & 106 & 105.8 & 14.5 & 23.3 & 17.2 & 3.7 & 31.3 & 26.4 & 5.9 & 111.3 & 106.8 & 16.6 \\
\hline SOMP & 14.9 & 12.9 & 1.2 & 23.1 & 23.1 & 2.1 & 114.1 & 107.3 & 22.5 & 23 & 16.8 & 0.4 & 32.9 & 26.8 & 4.7 & 113.9 & 107.4 & 21.4 \\
\hline
\end{tabular}

Table 3. Run-times (sec) of different algorithms for different density rates $(K(\%))$ in the presence of noise (input $\mathrm{SNR}=100 \mathrm{~dB}$, sampling rate $=25 \%, L=8$, and $K$ is known).

\begin{tabular}{cccc}
\hline $\boldsymbol{K}(\boldsymbol{\%})$ & $\mathbf{4}$ & $\mathbf{1 2}$ & $\mathbf{2 0}$ \\
\hline SIM & 0.017 & 0.027 & 0.027 \\
SOMP & 0.031 & 0.081 & 0.138 \\
\hline
\end{tabular}

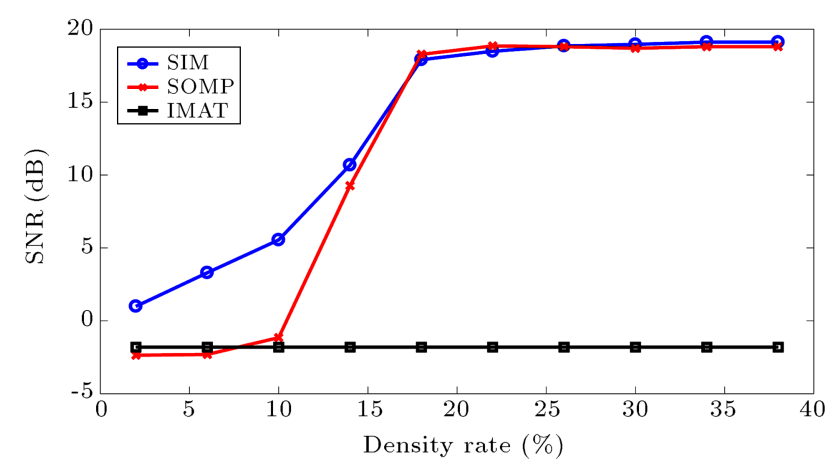

Figure 2. Reconstruction SNR versus number of signals (input $\mathrm{SNR}=20 \mathrm{~dB}$, sampling rate $=25 \%$, density rate $K=20 \%$, and the sparsity number is unknown).

Figure 2 shows the effect of the number of signals on the performance of SOMP and SIM. As observed in this figure, by increasing the number of signals, we get better results. Additionally, the SIM algorithm can reconstruct the signals better than the SOMP when the number of signals is small.

The reconstruction SNR for different sampling rates is depicted in Figure 3. According to this figure, the SNR values can be improved by increasing the sampling rate. Although the SOMP algorithm has better results for a small interval in the high sampling rates, SIM outperforms SOMP in most cases.

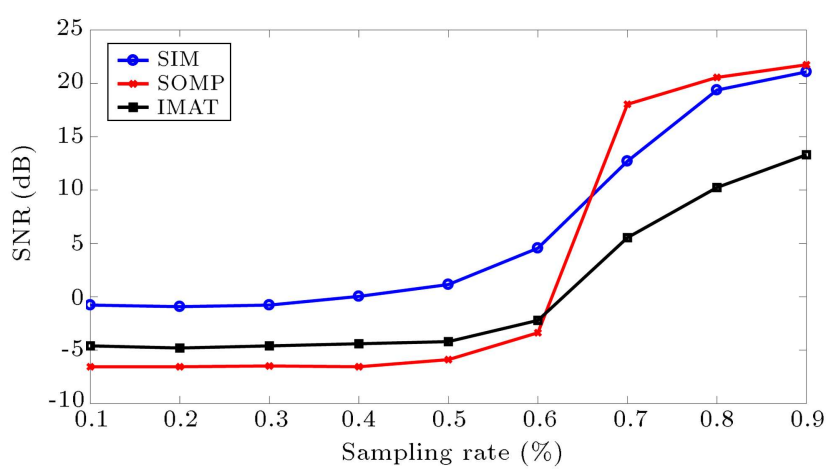

Figure 3. Reconstruction SNR versus sampling rate (input $\mathrm{SNR}=20 \mathrm{~dB}$, density rate $K=60 \%, L=8$, and the sparsity number is unknown).

Table 4 lists the SNRs of two reconstruction methods. Simulation results demonstrate the superiority of SIM over BIMAT in terms of the output SNR and the complexity. Therefore, SIM can be used instead of BIMAT in many applications such as distributed MIMO radar systems.

\section{Conclusion}

In this paper a novel method, namely SIM, was introduced for SSA problems. The proposed algorithm was an iterative method for the MMV models and its idea was that the summation of sparse vectors, sharing the same support, in the thresholding step of each iteration could enhance the probability of reconstruction. Indeed, the theoretical analysis with simulation results proved that SIM outperformed IMAT with respect to the SNR metric. We compared the proposed method with SOMP as a well-known algorithm in the MMV models. We concluded from the conducted numerical experiments that SIM was preferable in terms of SNR

Table 4. Reconstruction SNRs $(\mathrm{dB})$ of different algorithms for different density rates $(K(\%))$ in the presence of noise (input $\mathrm{SNR}=5 \mathrm{~dB}$, sampling rate $=20 \%, L=10$ ).

\begin{tabular}{ccccccc}
\hline $\boldsymbol{K}(\boldsymbol{\%})$ & $\mathbf{2 . 5}$ & $\mathbf{5}$ & $\mathbf{7 . 5}$ & $\mathbf{1 0}$ & $\mathbf{1 2 . 5}$ & $\mathbf{1 5}$ \\
\hline SIM & 14.1 & 11.6 & 8.8 & 6.7 & 4.9 & 3.3 \\
BIMAT & 13.3 & 9.8 & 7.5 & 6.0 & 4.1 & 3.4 \\
Improvement ratio (\%) & +6 & +18 & +17 & +12 & +19 & -2 \\
\hline
\end{tabular}


or SR, especially in noisier channels with low sampling rates and high density rates. Finally, it was observed that SIM was superior to BIMAT when complexity and efficiency were important factors.

\section{Acknowledgment}

The authors would like to thank Ehsan Asadi for his help in the analytical discussion subsection and Babak Barazandeh for introducing this topic to one of the authors.

\section{References}

1. Candès, E.J., Romberg, J., and Tao, T. "Robust uncertainty principles: Exact signal reconstruction from highly incomplete frequency information", IEEE Trans. of Inf. Theory, 52(2), pp. 489-509 (2006).

2. Donoho, D.L. "Compressed sensing", IEEE Trans. of Inf. Theory, 52(4), pp. 1289-1306 (2006).

3. Abbasi, H., Kavehvash, Z., and Shabany, M. "Improved CT image reconstruction through partial Fourier sampling", Sci. Iran., 23(6), pp. 2908-2916 (2016).

4. Amezquita-Sanchez, J. and Adeli, H. "Feature extraction and classification techniques for health monitoring of structures", Sci. Iran. A, Civ. Eng., 22(6), p. 1931 (2015).

5. Duarte, M.F., Cevher, V., and Baraniuk, R.G. "Model-based compressive sensing for signal ensembles", 47th Annu. Allerton Conf. on Commun., Control, and Comput., IEEE, pp. 244-250 (2009).

6. Gastpar, M. and Vetterli, M. "Source-channel communication in sensor networks", IPSN, Springer, pp. 162177 (2003).

7. Gorodnitsky, I.F., George, J.S., and. Rao, B.D. "Neuromagnetic source imaging with focuss: a recursive weighted minimum norm algorithm", Electroencephalogr. and Clin. Neurophysiol., 95(4), pp. 231-251 (1995).

8. Malioutov, D., Cetin M., and Willsky, A.S. "A sparse signal reconstruction perspective for source localization with sensor arrays", IEEE Trans. on Signal Process., 53(8), pp. 3010-3022 (2005).

9. Ibernon-Fernandez, R., Molina-Garcia-Pardo, J.M., and Juan-Llacer, L. "Comparison between measurements and simulations of conventional and distributed mimo system", IEEE Antennas and Wirel. Propag. Lett., 7, pp. 546-549 (2008).

10. Mohammadi, E., Fallah, A., and Marvasti, F. "Sampling and distortion tradeoffs for indirect source retrieval", IEEE Trans. on Inf. Theory, 63(11), pp. 6833-6848 (2017).

11. Tropp, J.A. and Gilbert, A.C. "Signal recovery from random measurements via orthogonal matching pursuit", IEEE Trans, on Inf. Theory, 53(12), pp. 46554666 (2007).
12. Determe, J.F., Louveaux, J., Jacques, L., and Horlin, F. "On the noise robustness of simultaneous orthogonal matching pursuit", IEEE Trans. on Signal Process., 65(4), pp. 864-875 (2017).

13. Tropp, J.A., Gilbert, A.C., and Strauss, M.J. "Algorithms for simultaneous sparse approximation. part i: Greedy pursuit", Signal Process., 86(3), pp. 572-588 (2006).

14. Rakotomamonjy, A. "Surveying and comparing simultaneous sparse approximation (or group-lasso) algorithms", Signal Process., 91(7), pp. 1505-1526 (2011).

15. Marvasti, F., Azghani, M., Imani, P., Pakrouh, P., Heydari, S.J., Golmohammadi, A., Kazerouni, A., and Khalili, M. "Sparse signal processing using iterative method with adaptive thresholding (IMAT)", 19th Int. Conf. on Telecommun. (ICT), IEEE, pp. 1-6 (2012).

16. Marvasti, F., Amini, A., Haddadi, F., Soltanolkotabi, M., Khalaj, B.H., Aldroubi, A., Sanei, S., and Chambers, J. "A unified approach to sparse signal processing", EURASIP J. on Adv. in Signal Process., $2012(1)$, p. 44 (2012).

17. Marvasti, F., Nonuniform sampling: theory and practice, Springer Science \& Business Media (2012).

18. Marvasti, F. and Mashadi, M.B. "Wideband analog to digital conversion by random or level crossing sampling", U.S. Patent 9,729,160 (2017).

19. Zarmehi, N. and Marvasti, F. "Sparse and low-rank recovery using adaptive thresholding", Digit. Signal Process., 73, pp. 145-152 (2018).

20. Azghani, M., Ghorbani A., and Marvasti, F. "Blind iterative nonlinear distortion compensation based on thresholding", IEEE Trans. on Circuits and Syst. II: Express Briefs, 64(7), pp. 852-856 (2017).

21. Mashhadi, M.B., Salarieh, N., Farahani, E.S., and Marvasti, F. "Level crossing speech sampling and its sparsity promoting reconstruction using an iterative method with adaptive thresholding", IET Signal Process., 11(6), pp. 721-726 (2017).

22. Abtahi, A., Azghani, M., Tayefi, J., and Marvasti, F. "Iterative block-sparse recovery method for distributed mimo radar", in Iran Workshop on Commun. and Inf. Theory (IWCIT), IEEE, pp. 1-4 (2016).

23. Zayyani, H., Babaie-Zadeh, M., and Jutten, C. "An iterative Bayesian algorithm for sparse component analysis in presence of noise", IEEE Trans. on Signal Process., 57(11), pp. 4378-4390 (2009).

24. Esmaeili, A., Kangarshahi, E.A., and Marvasti, F. "Iterative null space projection method with adaptive thresholding in sparse signal recovery", IET Signal Process, 12(5), pp. 605-612 (2018).

\section{Biographies}

Sahar Sadrizadeh received her BS degree in Electrical Engineering in 2015 and MS degree in Communications in 2017, both from Sharif University of 
Technology (SUT), Tehran, Iran. She started her $\mathrm{PhD}$ program in 2017 at the same university. She was a research intern at Hong-Kong University of Technology (HKUST) in summer 2015. Her research interests include sparse signal processing, image and multimedia processing, and applications of machine learning and deep learning in communication. Ms. Sadrizadeh ranked the third among more than 284,000 participants in the Nationwide University Entrance Exam for BS degree, in 2011. She also ranked the first amongst all the BS students of Sharif University of Technology in Electrical Engineering Department who started in 2011. Furthermore, she has been a member of the National Elite Foundation since 2011. She got direct admission to MS and PhD programs without national entrance examination as a privilege for excellent academic performance. She won the scholarship of Newton Foundation for PhD program.

Shahrzad Kianidehkordi received her BS degree in Electrical Engineering from Sharif University of Technology, Tehran, Iran, in 2017. She is currently pursuing her MS in Electrical Engineering at the University of Toronto. Her research interests include distributed computation, distributed machine learning algorithms, coding theory, and stochastic processes. She won Gold medal in National Mathematical Olympiad.

Mahdi Boloursaz Mashhadi received a dual-major BS degree (with honors) in Communications and Industrial Engineering in 2011, and MS and PhD degrees in Signal Processing in 2013 and 2018, all from Sharif University of Technology (SUT), Tehran, Iran. He has been a Research Associate with the ECE Department at the University of Central Florida (UCF), USA; Queens University, ON, Canada; and Sharif University of Technology, Tehran, Iran. His research interests encompass sparse signal processing; design and implementation of signal processing circuits; and image, speech, and multimedia processing. Dr. Boloursaz's awards include a best paper award at the EWDTS 2012 conference and several national, regional, and IEEE grants. He serves as a reviewer of IEEE transactions on signal processing, transactions on information theory, and signal processing letters.

Farokh Marvasti received his BS, MS, and PhD degrees all from Rensselaer Polytechnic Institute in 1970, 1971, and 1973, respectively. He has worked, consulted, and taught in various industries and academic institutions since 1972, among which are Bell Labs, University of California Davis, Illinois Institute of Technology, University of London, and King's College. He was one of the editors and Associate Editors of IEEE Trans on Communications and Signal Processing during 1990-1997. He has about 100 journal publications and has written several reference books; he has also several international patents. His last book was "Non-uniform Sampling: Theory and Practice" by Springer in 2001. He was also a guest editor of the Special Issue on Non-uniform Sampling for the Sampling Theory and Signal and Image Processing journal, May 2008. Besides being the co-founder of two international conferences (ICT's and SampTA's), he has been the organizer and special session chair of many IEEE conferences, including ICASSP. Recently, he was the lead editor of "Sparse Signal Processing" for the Special Issue of Eurasip Journal on Advances in Signal Processing. Dr. Marvasti is currently a Professor at Sharif University of Technology and the director of Advanced Communications Research Institute (ACRI). Also, he is the former head of the Center for MultiAccess Communications Systems. He spent his sabbatical leave with the Communications and Information Systems Group of University College London (UCL) in 2013. Professor Marvasti received a distinguished award from the Iranian Academy of Sciences in 2014 and a 5-year term chair position from Iranian National Science Foundation in 2015. He was also appointed as the Life Research Fellow by the IEEE, Iran Section, in 2018. 